\title{
Modelo de PRIORIZAÇÃo de INVESTIMENTOS EM SANEAMENTO BÁSICO UTILIZANDO PROGRAMAÇÃO LINEAR COM BASE EM INDICADORES AMBIENTAIS
}

\section{PRIORIZATION MODEL OF INVESTMENTS IN BASIC SANITATION USING LINEAR PROGRAMMING BASED ON ENVIRONMENTAL INDICATORS}

\author{
NAYRA VICENTE SOUSA DA SILVA \\ Engenheira Civil. Mestre em Engenharia Urbana pela Universidade Federal da Paraíba - UFPB \\ Roberto QUIRINO DO NASCIMENTO \\ Bacharel em Matemática. Doutor em Engenharia de Sistemas e Computação. Professor Adjunto da \\ Universidade Federal da Paraíba - UFPB
}

TARCISO CABRAL DA SILVA

Engenheiro Civil. Doutor em Engenharia Hidráulica. Professor Titular da Universidade Federal da Paraíba - UFPB

Recebido: 22/12/06 Aceito: 16/04/08

\begin{abstract}
RESUMO
Neste artigo apresenta-se uma contribuição referente ao desenvolvimento de um modelo de hierarquização de investimentos em saneamento. Utiliza-se a técnica da programação linear, com base em um indicador de salubridade ambiental, o ISA/JP1 (Silva, 2006). O objetivo é a maximização das condições de salubridade ambiental nas comunidades a serem beneficiadas. Dessa forma, procura-se intervir na alocação dos benefícios relativos às variáveis que compõem o subindicador correspondente ao saneamento básico. O modelo foi aplicado nas comunidades periurbanas localizadas na bacia do Rio Gramame, litoral sul do estado da Paraíba. Os benefícios propostos, segundo o modelo, resultariam em mudanças significativas, atingindo até a condição salubre, conforme os valores de investimentos disponíveis.
\end{abstract}

PALAVRAS-CHAVE: Priorização de investimentos, saneamento básico, salubridade ambiental, programação linear.

\begin{abstract}
In this article a contribution concerning the development of a hierarchization of investments in sanitation model is presented. The technique of the linear programming is used, based on an environmental health indicator, the ISA/JP1 (Silva, 2006). The aim is the maximization of the environmental health conditions in the communities to be benefited. In this way, it is attempted to interfere in the allocation of the benefits related to the subindicator variables corresponding to basic sanitation. The model was applied in five periurban communities of Lower Gramame River Basin, on the Southern Littoral of Paraiba State, Brazil. In this case, the proposed benefits would result in significant changes, even reaching the salubrious condition, according to values of available investments.
\end{abstract}

KEYWORDS: Priorization of investments, basic sanitation, environmental health, linear programming.

\section{INTRODUÇÃO}

Os investimentos em infra-estrutura para países em desenvolvimento sempre têm sido um problema de proporções consideráveis. Em particular, na área ambiental, estes não têm merecido a devida consideração frente às inversões em projetos para desenvolvimento econômico, sendo até às vezes conflitantes. Não raro, ocorrem investimentos em obras estruturais com repercussões negativas ao meio ambiente (Silva, 2006).

Em termos do meio ambiente urbano, no caso do Brasil, ainda com notórias deficiências em coberturas de sistemas e redes para o saneamento ambiental, os recursos para investimento não são abundantes, mesmo que sejam bastante conhecidas as repercussóes positivas na área de saúde pública. Neste contexto, a hierarquização de investimentos em saneamento ambiental se torna indispensável visando à maximização dos benefícios derivados.

Assim, a decisão acerca dos três questionamentos colocados por Teixeira e Heller (2001), "onde investir", "em que investir" e "quanto investir", deve ser fortalecida e tomada em bases racionais.
Não é raro no Brasil utilizar-se critérios empíricos e demandas políticas, nem sempre legítimas, para priorização de investimentos no setor de saneamento (Teixeira e Heller, 2001). Neste sentido, estes autores enfatizam a importância da sistematização de procedimentos metodológicos para o desenvolvimento de modelos de priorização de investimentos em saneamento, segundo indicadores de saúde.

Contudo, verifica-se uma carência de alternativas para atingir esta meta, sobretudo o estabelecimento de procedimentos adequados às condiçôes nacionais, capazes de embasar a cons- 
trução de modelos que desempenhem o papel de instrumento de auxilio à decisão sobre as prioridades de intervençôes para os serviços de saneamento.

Romera e Silva (2000) fazem importantes observaçôes sobre a necessidade de implementação de ferramentas para integração entre serviços ambientais e investimentos governamentais, que levem à implementação de políticas ambientais, objetivando a contribuição para um desenvolvimento ambientalmente sustentável. Propõem ainda uma metodologia baseada em indicadores ecológicos - econômicos através dos quais são atribuídas Notas de Enquadramento de Situação (NES) que permitem identificar cada Situação Real Local (SRL). Esta metodologia visa à classificação de situações para avaliação ambiental do uso da água por serviços urbanos de saneamento, considerando a integração de conteúdos ecológicos e econômicos nas escalas local e regional.

Teixeira e Heller (2001) desenvolveram um modelo de priorização de investimentos em saneamento, segundo critérios epidemiológico, sanitário, financeiro, social e ambiental, aplicando-o em 36 projetos da Companhia de Saneamento de Minas Gerais - COPASA/MG.

Posteriormente, Teixeira e Heller (2003) apresentaram um modelo mais simples de priorização de investimentos em saneamento, com ênfase em indicadores de saúde, construído a partir de uma combinação entre métodos de análise epidemiológica e financeira.

Neves e Neves (2003) propóem um procedimento metodológico para a seleção ou hierarquização de projetos de saneamento. O foco da proposta para a seleção de projetos é a condição de saúde da população a ser beneficiada. A proposta se insere no campo da análise custo-eficácia, e segue esta lógica baseando-se na relação entre benefícios, medidos através de um indicador, e o custo medido monetariamente. Para isso sugerem um indicador denominado DALY - Disability Adjusted Life-Years, desenvolvido para a área de saúde pública (Murray, 1996 apud Neves e Neves, 2003).

Alguns critérios de priorização podem ser encontrados em textos de relatórios de estudos e projetos para intervenções em saneamento básico. Baseiamse normalmente em critérios ambientais, financeiros, sociais e epidemiológicos.
A Fundação Nacional de Saúde FUNASA do Ministério da Saúde através da Portaria no 151 , de 20/02/2006 (BrasilL, 2006), em seu art. 3º, estabelece que os critérios de prioridade são essencialmente indicativos, devendo a FUNASA, quando da priorização das ações, observar as condições específicas da execução dos projetos, a sustentabilidade, variação dos indicadores de saúde e outras questóes relativas à viabilidade técnica dos projetos apresentados e o interesse público.

Os critérios e procedimentos básicos estabelecidos pela FUNASA são fundamentados em dados de saneamento básico e indicadores de saúde que visam ampliar e aprimorar os parâmetros de atuação da Instituição nas ações de saneamento. Buscam maior eficiência na aplicação de recursos financeiros e maiores impactos das ações na qualidade de vida e de saúde da população brasileira. Ainda na Portaria no ${ }^{\circ} 151 / 2006$, as ações de saneamento seguem as diretrizes definidas pela Resolução $n^{\circ} 322 / 03$ do Conselho Nacional de Saúde e a atuação será, prioritariamente, em municípios com população de até 30 mil habitantes, observando critérios epidemiológico e sanitário, com baixo Índice de Desenvolvimento Humano (IDH), bem como aqueles definidos como prioritários pelo Programa Fome Zero do Governo Federal.

Neste trabalho apresenta-se uma contribuição direcionada à obtenção de procedimentos metodológicos adequados para a priorização de investimentos em saneamento básico, com base em um indicador de salubridade ambiental. O objetivo é otimizar as condições de salubridade ambiental de agrupamentos populacionais não convencionais, tais como ocupações espontâneas e comunidades periurbanas, bem como auxiliar na tomada de decisão quanto aos investimentos, com base na abrangência das variáveis indicadoras que compõem os indicadores de salubridade ambiental, o ISA/JP1, e o de saneamento básico, I, definidos por Silva (2006). A contribuição referese a um modelo de hierarquização de investimentos em saneamento básico, denominado MPIS/PL, que utiliza a técnica da Programação Linear.

Foi feita uma aplicação nas comunidades periurbanas de Gramame, Engenho Velho, Colinas do Sul, Mumbaba de Baixo e Mituaçu, localizadas na bacia hidrográfica do baixo curso do rio Gramame, litoral sul do estado da Paraíba.

\section{METODOLOGIA}

\section{O modelo ISA/JP I}

A metodologia deste trabalho envolve a proposição de um procedimento baseado em um indicador de salubridade ambiental, o ISA/JP1 (Silva, 2006), este adaptado do modelo ISA/JP (Batista e Silva, 2006). Por sua vez, o ISA/JP foi modificado a partir do modelo ISA desenvolvido pelo Conselho Estadual de Saneamento do Estado de São Paulo (São Paulo, 1999), ao qual foi incorporado o subindicador relativo à drenagem urbana.

Na verdade, o ISA/JP1 agrega mais um subindicador específico, o de condições de moradia, Icm, à semelhança do modelo ISA/OE desenvolvido por Dias, Borja e Moraes (2004).

A composição do ISA/JP1 é dada pela média ponderada de subindicadores específicos, com avaliação de atributos não apenas quantitativos, mas também qualitativos, e da condição da gestão dos sistemas pertinentes. Sua composição é dada conforme Equação (1) e seu valor pode variar de 0 a 1 , assim como os valores dos subindicadores Iab, Ies, etc, descritos adiante.

ISA $/ \mathrm{JP} 1=0,20 \mathrm{Iab}+0,20 \mathrm{Ies}+$

$+0,15 \mathrm{Irs}+0,10 \mathrm{Icv}+0,10 \mathrm{Irh}+$

$+0,10 \mathrm{Idu}+0,10 \mathrm{Icm}+0,05$ Ise

Onde:

Iab - Subindicador de Abastecimento de Água;

Ies - Subindicador de Esgotos Sanitários;

Irs - Subindicador de Resíduos Sólidos;

Icv - Subindicador de Controle de Vetores;

Irh - Subindicador de Recursos Hídricos;

Idu - Subindicador de Drenagem Urbana;

Icm - Subindicador de Condiçôes de Moradia;

Ise - Subindicador Socioeconômico.

$\mathrm{Na}$ formulação do modelo ISA/JP1 propõe-se também uma classificação para avaliação da performance da salubridade ambiental, em complemento à mensuração numérica. São sugeridas faixas numéricas de classificação às quais se atribuem situações de salubridade ambiental. Esta pro- 
posta de classificação da performance da salubridade ambiental foi adaptada de Batista e Silva (2006), na qual se inseriu uma nova situação, de Salubridade Aceitável, conforme apresentado na Tabela 1. Silva (2006) propôs esta nova faixa de classificação e justifica devido ao amplo intervalo da situação Salubre apresentada no modelo ISA/JP. Assim, para restringir o alcance da situação Salubre foi reduzido seu intervalo, incorporando a nova faixa já mencionada.

\section{Indicador de saneamento básico (I)}

A metodologia referida a seguir trata do desenvolvimento de um modelo para a alocação de benefícios em saneamento básico, considerando a priorização das possíveis implantaçôes dos investimentos com as quais a função objetivo, correspondente ao subindicador relativo ao saneamento básico, é maximizada, obedecendo-se às restriçōes do problema.

Com este intuito, foram definidos como os benefícios passíveis de investimentos os relativos ao saneamento básico, conforme a Lei no $11.445 / 07$, que estabelece diretrizes nacionais para o saneamento básico e para a sua política federal (Brasil, 2007), compreendendo: abastecimento d'água, esgotamento sanitário, limpeza urbana e manejo de resíduos sólidos, além dos serviços de drenagem e de manejo de águas pluviais.

Justifica-se tal eleição ao fato de que no modelo ISA/JP1, as variáveis subindicadoras relativas ao saneamento básico são contempladas pelos maiores coeficientes lineares, ou seja, um pequeno acréscimo em pelo menos uma destas variáveis produzirá um maior incremento no ISA/JP1. Ademais, são benefícios passíveis de implantação imediata e com maiores impactos na salubridade ambiental, tão logo sejam implantados estes projetos. Ou seja, após a devida colocação em operação, geram imediatamente impactos positivos, diferentemente dos relativos aos temas das outras variáveis que são de respostas lentas ou de aplicação distribuída. Assim, ações de melhoria de renda, por exemplo, são certamente intervenções com respostas obtidas de médio a longo prazo, após o início da adoção de uma política de melhoria e distribuição de renda.

Tabela I - Situação de salubridade ambiental por faixa de situação (\%)

\begin{tabular}{cc}
\hline Situação da salubridade & Pontuação do ISA/JP1 \\
\hline Insalubre (INS) & $0-25,50$ \\
Baixa salubridade (BSB) & $25,51-50,50$ \\
Média salubridade (MSB) & $50,51-75,50$ \\
Salubridade Aceitável (SAC) & $75,51-90,00$ \\
Salubre (SAL) & $90,01-100,00$ \\
\hline
\end{tabular}

O indicador ISA/JP1, no formato aqui explicitado, trata-se de uma combinação linear de variáveis ou subindicadores representantes da natureza dos subcomponentes. Assim, o ISA/JP1 pode ser expresso como uma função " $f$ definida como apresentada na Equação 2.

ISA/JP1 = $f$ (Iab, Ies, Irs, Idu, Icv, Irh, Icm, Ise)

Neste formato, nota-se que as quatro primeiras variáveis independentes representam os subindicadores referentes ao saneamento básico. As outras variáveis, embora evidentemente contribuam positivamente com o indicador ISA/JP1, não provocam impactos pelo menos semelhante, ao receberem iguais incrementos.

É evidente que uma melhoria nas condições de saneamento básico implicará em melhorias em outros aspectos da salubridade ambiental, como os de saúde pública, representadas pelo indicador Icv.

$\mathrm{O}$ indicador ISA/JP1 pode ser entendido como uma soma de dois outros indicadores, sendo um referente ao saneamento básico, e o outro referente às variáveis complementares ou descritivas das condiçôes de saúde pública, habitacionais, socioeconômicas e recursos hídricos. $\mathrm{Na}$ verdade, este último indicador refere-se à oferta de recursos hídricos e medidas administrativas (não estruturais).

Destarte, pode-se escrever o indicador de salubridade ambiental, ISA/JP1, como se segue:

ISA $/ \mathrm{JP} 1=\mathrm{I}+\mathrm{Ic}$

Onde I é o Indicador de Saneamento Básico, na forma:

$\mathrm{I}=0,20 \mathrm{Iab}+0,20 \mathrm{Ies}+0,15 \mathrm{Irs}+0,10 \mathrm{Idu}$

e Ic é o Indicador de Variáveis Complementares,

$\mathrm{Ic}=0,10 \mathrm{Icv}+0,10 \mathrm{Irh}+$

$+0,10 \mathrm{Icm}+0,05$ Ise

\section{Modelo de hierarquização de investimentos em saneamento com base no ISA/JPI e na técnica da Programação Linear}

A metodologia agora empregada para otimizar o ISA/JP1, priorizando benefícios nas comunidades segundo critérios pré-definidos apresentados a seguir, abrange a utilização de um modelo matemático de programação linear $(\mathrm{PL})$.

A estrutura do modelo desenvolvido, denominado MPIS/PL, é constituída de uma função objetivo e de um conjunto de restriçôes, que fazem referência às variáveis do problema. Conforme Prado (1999), a estratégia da PL é resolver o problema de otimização através da transformação das características do problema em um modelo abstrato matemático, que nada mais é do que um conjunto de equaçôes matemáticas.

De uma forma sucinta, um problema de programação linear trata de otimização onde a função objetivo é linear e as restrições são inequaçôes ou equações lineares. Na forma de programação linear inteira mista, refere-se a problemas onde as variáveis podem ser contínuas ou discretas.

O ISA/JP1 é utilizado lançandose mão de um modelo de programação linear para as variáveis subindicadores de abastecimento de água, esgotamento sanitário, coleta de resíduos sólidos e drenagem urbana. Este conjunto de subindicadores compõe a variável auxiliar I, já definida na Equação (4).

Com relação à avaliação da performance da salubridade ambiental, no caso referente à parcela correspondente aos serviços de saneamento básico, constam na Tabela 2 os valores das faixas de situação para o ISA/JP1 e do I, sendo que a última coluna foi estabelecida através de um percentual relativo das faixas de situação do ISA/JP1. 
Tabela 2 - Enquadramento das situações relativas às faixas de performance da salubridade ambiental (ISA/JPI) e do saneamento básico (I) Pontuação

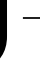

Insalubre (INS)

Baixa salubridade (BSB)

Média salubridade (MSB)

Salubridade Aceitável (SAC)

Salubre (SAL)

)

Situação da salubridade

\begin{tabular}{cc}
\multicolumn{2}{c}{ Pontuação } \\
ISA/JP1 & I \\
\hline $0,00-25,50$ & $0,00-16,57$ \\
$25,51-50,50$ & $16,58-32,82$ \\
$50,51-75,50$ & $32,83-49,07$ \\
$75,51-90,00$ & $49,08-58,50$ \\
$90,01-100,00$ & $58,51-65,00$ \\
\hline
\end{tabular}

O modelo de programação linear procura maximizar o indicador ISA/JP1 da cidade, do bairro, do setor ou comunidade, considerando um conjunto de variáveis restritivas de naturezas diversas.

As restriçõos consideradas são: um valor mínimo aceitável adotado para o ISA/JP1, os valores das intervenções não alocáveis (no caso de pré-existência) nas comunidades obtidos com a aplicação do ISA/JP1; os valores financeiros destes possíveis investimentos; e os pesos relativos das variáveis subindicadoras do indicador de saneamento básico (I). Uma outra restrição é a referente à lógica de implantação de projetos de saneamento: projeto de esgotamento sanitário somente deverá ser alocado caso já exista sistema de abastecimento de água ou sejam conjuntamente alocados.

Para resolvê-lo, o problema foi estruturado com o cumprimento das seguintes etapas:

I) Definiçãao das variáveis do problema;

II) Definição da função objetivo;

III) Definição do conjunto de restriçōes.

A estrutura do modelo é mostrada detalhadamente a seguir:

I) Definição das variáveis do problema

As variáveis consideradas são as do saneamento básico, referidas ao conjunto que compreende os benefícios: abastecimento de água, o esgotamento sanitário, o manejo de resíduos sólidos e das águas pluviais.

Assim, tem-se:

$\mathrm{A}=(1,2, \ldots \mathrm{k})$ um conjunto de comunidades (índice j) ou centros urbanos considerados para a recepção dos "s" benefícios.
$\mathrm{B}=(1,2, \ldots \mathrm{s})$ um conjunto dos benefícios (índice i) possíveis de serem implantados nas " $\mathrm{k}$ " comunidades. Onde:

$\mathrm{I}_{\mathrm{ij}}=*^{1}$, se o benefício "i" for alocado na comunidade "j"

$I_{j}=$ I da comunidade “ $j$ ”

No qual:

$\mathrm{I}_{\mathrm{ij}}$ - Subindicador referente ao benefício

"i" alocado na comunidade "i”";

I. - I (Indicador de Saneamento Básico) da comunidade " $j$ ".

II) Definição da função objetivo Objetivo:

Maximizar I priorizando as comunidades mais populosas.

Função Objetivo:

$F=\sum_{j=1}^{k} p_{j} I_{j}$

$\mathrm{Na}$ qual tem-se:

$\mathrm{p}_{\mathrm{j}}=\frac{\mathrm{n}_{\mathrm{j}}}{\mathrm{k}^{\mathrm{k}} \mathrm{n}_{\mathrm{j}}}$

Onde:

$\mathrm{n}_{\mathrm{j}}$ - População da comunidade “ $j$ ”;

$p_{j}^{\prime}$ - Coeficiente da comunidade " $j$ "

relativo à população total;

$k$ - número de comunidades consideradas no problema. restriçōes

III) Definição do conjunto de

$1^{\text {a }}$ Restrição:

O custo total da implantação dos benefícios deve ser no máximo um valor fixado.

$C=!_{i=1}^{5} \stackrel{k}{j=1}_{j i j} C_{i j}$

Onde:

Cij - Custo de implantação do benefício "i” na comunidade “j”;

$s$ - número de benefícios considerados no problema. 2a Restrição:

As intervenções devem proporcionar, no mínimo, um estado de Média Salubridade a cada comunidade.

$\mathrm{I}_{\mathrm{j}} \geq 0,328 \quad \mathrm{j}=1,2, . ., \mathrm{k}$

$\mathrm{O}$ valor mínimo adotado para o $I_{j}$ considerado é equivalente à soma dos pesos das variáveis de saneamento básico incidindo sobre o valor da faixa de enquadramento com Média Salubridade.

3a Restrição:

Composição do I:

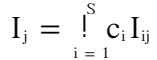

Onde:

$c_{\text {. }}$ - Peso relativo à importância do benefício " $i$ " incidente nas variáveis subindicadoras.

Deve ser observado que o $I_{i j}$ assume o valor da variável auxiliar $W_{i j}$ nos casos em que já existe uma condição aceitável (onde o sistema já está implantado com pelo menos um dos benefícios), refletindo assim nos valores das variáveis subindicadoras referentes aos benefícios já implantados.

4a Restrição:

Quando a variável $I_{i j}$ assumir valor da variável auxiliar, $W_{i j}$, as frações dos benefícios já instalados devem ter suas participações na função $I$.

$\mathrm{W}_{\mathrm{ij}}=\mathrm{f}_{\mathrm{ij}} \mathrm{I}_{\mathrm{ij}}$

Onde:

$f_{i j}$-Fração do benefício “ $i$ ” já instalado na comunidade " $j$ ".

5a Restrição:

Para esta restrição foi estabelecido que apenas as comunidades em que existe abastecimento de água poderão ser contempladas com sistema de esgotamento sanitário.

$\mathrm{I}_{\mathrm{ij}} \geq \mathrm{I}_{2 \mathrm{j}} \quad \mathrm{j}=1,2 \ldots, \mathrm{k}$

Onde:

$\mathrm{I}_{1 \mathrm{j}}$ - Indicador de Abastecimento de Âgua na comunidade “ $i$ ";

$\mathrm{I}_{2 \mathrm{j}}$ - Indicador de Esgotamento Sanitário na comunidade " $j$ ".

\section{Área de aplicação - características}

As localidades contempladas neste estudo são as comunidades periurbanas do Baixo Curso do Rio Gramame, sendo elas Gramame, Engenho Velho, Colinas do Sul, e Mumbaba de Baixo pertencentes ao município de João Pessoa, e Mituaçu situado no município do Conde no litoral Sul do Estado da Paraíba. 
Estas comunidades estão inseridas em zona rural, caracterizadas pela presença de atividades agrícola e/ou pecuária nos seus entornos e pelos costumes e cultura de seus moradores, apesar da proximidade da malha urbana das cidades de João Pessoa e Conde.

Formam aglomerações com características típicas de comunidades periféricas dos centros urbanos, reflexos da atual política econômica e social do Brasil, focada no desenvolvimento excludente, sob os aspectos da má distribuição de renda e da estrutura fundiária. As populações das comunidades totalizaram 7.334 habitantes na ocasião dos levantamentos dos dados feitos em agosto de 2005.

Acrescida a estas realidades observam-se ainda outras peculiaridades inerentes a estas comunidades, resumidas a seguir: i) Ocupação territorial em geral feita de forma espontânea; ii) Comunidades com baixa renda; iii) Maioria de empregos informais e temporários; iv) Baixo grau de escolaridade; v) Precariedade de serviços públicos e infra-estrutura.

\section{Aplicação do modelo MPIS/ṔL}

A aplicação do modelo MPIS/PL, desenvolvido por Silva (2006), permite a otimização do ISA/JP1, alocando-se os benefícios que compõem a variável auxiliar I, considerando para isto o custo de cada intervenção, além de um conjunto de variáveis restritivas e priorizando as comunidades mais populosas.

Utilizou-se o modelo para diversos percentuais do investimento $(5 \%, 10 \%$, 20\%, 30\% 40\%, 50\%, 60\% 70\%, 80\% e $100 \%$ ) relativos ao custo total de implantação das 4 intervençóes propostas referentes ao saneamento básico. As respostas de alocação definiam totais de investimentos a serem alocados, além de resíduos ou diferenças. Por exemplo, informando-se no programa $5 \%$ do valor total, ter-se-ia apenas o percentual de $4,86 \%$ referente à soma dos valores correspondentes aos benefícios alocados.

Para cada percentual de investimento informado, geraram-se cenários diferenciados de alocação de benefícios e de valores de I, no qual a partir destes foi possível calcular um ISA/JP1 melhorado considerando estas intervenções. Desta forma, foi possível analisar a performance da salubridade de cada comunidade segundo cada resultado obtido.

Detalha-se a seguir a estrutura do modelo utilizado para a aplicação nas comunidades da Bacia do Baixo Gramame:
1) Maximize $F=!_{j=1}^{5} p_{j} I_{j}$

II) Sujeito a:

$!_{\mathrm{i}=1 \mathrm{j}=1}^{4} \mathrm{C}_{\mathrm{ij}} \mathrm{I}_{\mathrm{ij}} \leq$ Custo $(5 \%, 10 \%$,

$20 \%, \ldots 100 \%$ do total para investimento)

$\mathrm{I}_{\mathrm{j}} \geq 0,328 \mathrm{j}=1,2 \ldots 5$

$I_{j}-!_{j=1}^{4} c_{i} I_{i j}=0$

$\mathrm{W}_{\mathrm{ij}}=\mathrm{f}_{\mathrm{ij}} \mathrm{I}_{\mathrm{ij}}$

$I_{\mathrm{ij}} \geq \mathrm{I}_{2 \mathrm{j}}=1,2, \ldots, 5$

$\mathrm{I}_{\mathrm{j}} \geq 0, \mathrm{~W}_{\mathrm{ij}} \geq 0, \mathrm{I}_{\mathrm{ij}}=1$

$\mathrm{O}$ valor de $\mathrm{I}_{j}$ considerado deve assumir o valor mínimo de 0,5051 $(0,328=0,65 \times 0505)$, ou seja, equivalendo à Média Salubridade com relação ao saneamento básico.

O modelo descrito foi aplicado nas 5 (cinco) comunidades da Bacia do Baixo Gramame. A Tabela 3 mostra a descrição das variáveis consideradas no problema.

Os valores dos investimentos de cada benefício passível de implantação nas comunidades constam na Tabela 4 .

\section{RESULTADOS E DISCUSSÃO}

Os resultados do modelo ISA/JP1, abrangendo os valores da salubridade

Tabela 3 - Descrição das variáveis do problema consideradas no modelo

\begin{tabular}{cccc}
\hline Índice (i) & Benefícios ou variáveis subindicadoras (Ii) & Índice (j) & Comunidades \\
\hline 1 & Abastecimento D’Água (SAA) & 1 & Mumbaba de Baixo \\
2 & Esgotamento Sanitário (SES) & 2 & Gramame \\
3 & Coleta, Tratamento e Disposição Final dos & 3 & Mituaçu \\
& Resíduos Sólidos (SRS) & & Colinas do Sul \\
4 & Drenagem das Águas Pluviais (DU) & 4 & Engenho Velho \\
\hline
\end{tabular}

Tabela 4 - Resumo dos valores dos investimentos referentes aos diversos benefícios alocáveis nas comunidades

\begin{tabular}{cccccc}
\hline Comunidades & \multicolumn{3}{c}{ Benefícios alocáveis $(\mathrm{R} \$)$} & Total \\
& SAA & SES & SRS & DU & da comunidade $(\mathrm{R} \$)$ \\
\hline Mumbaba de Baixo & $0,00^{(1)}$ & $134.889,00$ & $0,00^{(1)}$ & $355.747,00$ & $490.636,00$ \\
Gramame & $0,00^{(1)}$ & $49.818,00$ & $0,00^{(1)}$ & $480.210,00$ & $530.028,00$ \\
Mituaçu & $141.740,00$ & $90.391,00$ & $45.534,00$ & $555.547,00$ & $833.212,00$ \\
Colinas do Sul & $0,00^{(1)}$ & $1.064 .828,00$ & $0,00^{(1)}$ & $3.297 .763,00$ & $4.362 .591,00$ \\
Engenho Velho & $173.400,00$ & $96.080,00$ & $32.176,00$ & $952.880,00$ & $1.254 .591,00$ \\
Total & $315.140,00$ & $1.436 .006,00$ & $77.710,00$ & $5.642 .147,00$ & $7.471 .003,00$ \\
\hline
\end{tabular}

(1) Benefício já existente na comunidade 
ambiental e do indicador de saneamento básico I, aplicados às comunidades estudadas estão mostrados na Tabela 5.

A aplicação do Modelo de Hierarquização de Investimentos em Saneamento, com base no ISA/JP1 e utilizando a técnica da Programação Linear - MPIS/PL, às cinco comunidades gerou 10 cenários de investimentos sendo 5 deles mostrados nas Tabelas 6 a 10. Nestas são vistas as comunidades a serem beneficiadas, os benefícios priorizados $(\mathrm{S})$ ou não $(\mathrm{N})$, ou pré-existentes $(\mathrm{E})$ além dos valores de ISA/JP1, as situações de salubridade na conjuntura atual e considerando a implantação do benefício, além dos valores dos investimentos. $\mathrm{O}$ modelo responde às alocaçôes de benefícios para as 5 comunidades como um conjunto. Os valores de investimentos alocados são calculados como percentuais do valor total equivalente à soma de todos os investimentos possíveis, referentes à implantação de projetos de saneamento básico nas comunidades.

Conforme mostra a Tabela 6, com $\mathrm{R} \$ 347.316,00$ disponíveis para investimento, observa-se que seriam alocados apenas os SAA nas comunidades de Mituaçu e Engenho Velho, e SRS na comunidade de Engenho Velho. Nesta última comunidade, com a implantação

Tabela 5 -População, valores dos subindicadores primários, do ISA/JPI, do I em \% e das respectivas situações da salubridade de cada comunidade

\begin{tabular}{|c|c|c|c|c|c|c|c|c|c|c|}
\hline \multirow[t]{3}{*}{ População } & $\mathrm{Iab}$ & Ies & Irs & Icv & Irh & Idu & $\mathrm{Icm}$ & Ise & Valor & $\begin{array}{l}\text { Situação da } \\
\text { salubridade }\end{array}$ \\
\hline & & & & & & & & & ISA/JP1 & ISA/JP1 \\
\hline & & & & & & & & & I & I \\
\hline \multirow[t]{2}{*}{1.231} & 93,33 & 0,00 & 100,00 & 56,25 & 70,00 & 11,95 & 64,7 & 24,21 & 55,17 & MSB \\
\hline & & & & & & & & & 34,86 & MSB \\
\hline \multirow[t]{2}{*}{450} & 91,67 & 0,00 & 100,00 & 18,75 & 70,00 & 8,52 & 65,11 & 70,80 & 53,11 & MSB \\
\hline & & & & & & & & & 34,18 & MSB \\
\hline \multirow[t]{2}{*}{854} & 33,33 & 0,00 & 0,00 & 50,00 & 33,33 & 14,23 & 58,29 & 51,92 & 24,85 & INS \\
\hline & & & & & & & & & 8,09 & INS \\
\hline \multirow[t]{2}{*}{3.935} & 93,33 & 0,00 & 100,00 & 18,75 & 70,00 & 15,99 & 68,09 & 60,62 & 53,98 & MSB \\
\hline & & & & & & & & & 35,27 & MSB \\
\hline \multirow[t]{2}{*}{864} & 33,33 & 0,00 & 0,00 & 56,25 & 93,33 & 15,35 & 64,73 & 43,49 & 31,81 & BSB \\
\hline & & & & & & & & & 8,20 & INS \\
\hline
\end{tabular}

(1) INS - Insalubre; BSB - Baixa Salubridade; MSB - Média Salubridade.

Tabela 6 - Resultados obtidos para a alocação de investimentos de 4,65\% do valor total, $R \$ 347.3$ I 6,00; valores do ISA/JP I e I atuais e segundo a alocação dos benefícios priorizados propostos de acordo com o modelo MPIS/PL, com as respectivas situações de salubridade

\begin{tabular}{|c|c|c|c|c|c|c|c|c|}
\hline \multirow[t]{4}{*}{ Comunidades } & \multicolumn{2}{|c|}{ Atual } & \multicolumn{4}{|c|}{ Benefícios priorizados } & \multicolumn{2}{|c|}{ Proposta } \\
\hline & Valor & $\begin{array}{l}\text { Situação da } \\
\text { salubridade }\end{array}$ & SAA & SES & SRS & DU & Valor & $\begin{array}{l}\text { Situação da } \\
\text { salubridade }\end{array}$ \\
\hline & ISA/JP1 & ISA/JP1 & & & & & ISA/JP1 & ISA/JP1 \\
\hline & I & I & & & & & I & I \\
\hline \multirow[t]{2}{*}{ Mumbaba de Baixo } & 55,17 & MSB & $\mathrm{E}$ & $\mathrm{N}$ & $\mathrm{E}$ & $\mathrm{N}$ & 55,17 & MSB \\
\hline & 34,86 & MSB & & & & & 34,86 & MSB \\
\hline \multirow[t]{2}{*}{ Gramame } & 53,11 & MSB & $\mathrm{E}$ & $\mathrm{N}$ & $\mathrm{E}$ & $\mathrm{N}$ & 53,11 & MSB \\
\hline & 34,18 & MSB & & & & & 34,18 & MSB \\
\hline \multirow[t]{2}{*}{ Mituaçu } & 24,85 & INS & $S$ & $\mathrm{~N}$ & $\mathrm{~N}$ & $\mathrm{~N}$ & 38,18 & BSB \\
\hline & 8,09 & INS & & & & & 21,42 & BSB \\
\hline \multirow[t]{2}{*}{ Colinas do Sul } & 53,98 & MSB & $\mathrm{E}$ & $\mathrm{N}$ & $\mathrm{E}$ & $\mathrm{N}$ & 53,98 & MSB \\
\hline & 35,27 & MSB & & & & & 35,265 & MSB \\
\hline \multirow[t]{2}{*}{ Engenho Velho } & 31,81 & BSB & $S$ & $\mathrm{~N}$ & $S$ & $\mathrm{~N}$ & 60,14 & MSB \\
\hline & 8,20 & INS & & & & & 36,53 & MSB \\
\hline
\end{tabular}


destes dois benefícios, simultaneamente, seriam geradas melhorias de 28,33\% nos valores do ISA/JP1 e no I, circunstância que permitiria a comunidade sair de uma situação de Baixa Salubridade para a Média Salubridade na Salubridade Ambiental e de Insalubre para Média Salubridade no que se refere ao Saneamento Básico. Na comunidade de Mituaçu também ocorreria uma melhoria, porém não tão significativa se comparada a Engenho Velho. Seus valores do ISA/JP1 e de I aumentariam em pouco mais de $13 \%$ e suas situaçóes de salubridade passariam da condição Insalubre para de Baixa Salubridade.

Com R\$ 1.119.775,00 de recursos disponíveis para investimento (Tabela 7) alocar-se-iam SAA e SRS em todas as comunidades que não contam com estes benefícios; SES em quase todas as comunidades, com exceção de Colinas do Sul; e por último a DU apenas em Mumbaba de Baixo. Mumbaba de Baixo seria a única comunidade que para este valor de investimento, seriam alocados todos os benefícios que se apresentavam ausentes na mesma, com evolução nos indicadores de $28,80 \%$, saindo de Média Salubridade para Salubridade Aceitável no ISA/JP1 e para Salubre no I. Contudo, em Engenho Velho e Mituaçu ocorreria uma evolução maior nos valores dos indicadores, de 48,33\%. Como resultado desta melhoria em ambas, Mituaçu passaria da situação de salubridade ambiental Insalubre para Média Salubridade; e I passaria à condição de Salubridade Aceitável. Engenho Velho evoluiria para a condição de Salubridade Aceitável em ambos os indicadores a partir das condiçōes de Baixa Salubridade no ISA/JP1 e Insalubre no I. Colinas do Sul seria a única sem benefício alocado.

Assim como se apresenta na Tabela 8 , a possível disponibilidade de $\mathrm{R} \$ 2.740 .150,00$ para investimento nas comunidades permitiria que em Mumbaba de Baixo e Mituaçu fossem alocados todos os benefícios os quais estas comunidades não contavam; na primeira foram alocados SES+DU; e na segunda comunidade SAA+SES+SRS+DU.

Este panorama de investimentos proporcionaria grandes evoluções nos valores dos indicadores e no estado de salubridade: Mumbaba de Baixo sairia de uma situação de Média para Salubridade Aceitável no ISA/JP1 e para Salubre relativo ao I; Mituaçu sairia da condição Insalubre para a Salubridade Aceitável no ISA/JP1 e para Salubre relativo ao I, aparecendo com uma evolução ainda mais positiva em relação a Engenho Velho. Em Engenho Velho a alocação de SAA+SES+SRS também proporcionaria melhorias bastante significativas, elevando os indicadores em 49,33\%, e uma situação de Baixa Salubridade para a Salubridade Aceitável no ISA/JP1, ao passo que no I a situação passaria de Insalubre para Salubridade Aceitável . Em Colinas do Sul e Gramame seriam alocados apenas SES e ambas continuaram na mesma situação de salubridade ambiental antes da intervenção, de Média Salubridade, à medida que o novo I proporciona a condição de Salubridade Aceitável.

Segundo se apresenta na a Tabela 9, observa-se que Mituaçu e Engenho Velho seriam as únicas que não teriam o benefício DU alocado. Gramame, assim como Mumbaba de Baixo e Colinas do Sul, seriam contemplados com SES + DU.

Os benefícios que seriam investidos nestas comunidades proporcionariam um progresso significativo nos valores dos indicadores ISA/JP1 e I e evidentemente na situação de salubridade. Mumbaba de Baixo, Gramame, Colinas do Sul e Engenho Velho atingiriam valores do ISA/JP1 e I maiores do que $80 \%$ e $56,53 \%$ respectivamente, evolucionando assim para a classificação de Salubridade Aceitável ou Salubre.

Mituaçu seria a única a enquadrarse na situação de Média Salubridade quanto à salubridade ambiental após o

Tabela 7 - Resultados obtidos para a alocação de investimentos de |4,99\% do valor total, R\$ I I | 9.775,00; valores do ISA/JPI e I atuais e segundo a alocação dos benefícios priorizados propostos de acordo com o modelo MPIS/PL, com as respectivas situações de salubridade

\begin{tabular}{|c|c|c|c|c|c|c|c|c|}
\hline \multirow[t]{4}{*}{ Comunidades } & \multicolumn{2}{|c|}{ Atual } & \multicolumn{4}{|c|}{ Benefícios priorizados } & \multicolumn{2}{|c|}{ Proposta } \\
\hline & Valor & $\begin{array}{l}\text { Situação da } \\
\text { salubridade }\end{array}$ & SAA & SES & SRS & DU & Valor & $\begin{array}{l}\text { Situação da } \\
\text { Salubridade }\end{array}$ \\
\hline & ISA/JP1 & ISA/JP1 & & & & & ISA/JP1 & ISA/JP1 \\
\hline & I & I & & & & & I & I \\
\hline \multirow[t]{2}{*}{ Mumbaba de Baixo } & 55,17 & MSB & $\mathrm{E}$ & $S$ & $\mathrm{E}$ & $S$ & 83,97 & SAC \\
\hline & 34,86 & MSB & & & & & 63,66 & SAL \\
\hline \multirow[t]{2}{*}{ Gramame } & 53,11 & MSB & $\mathrm{E}$ & $S$ & $\mathrm{E}$ & $\mathrm{N}$ & 73,11 & MSB \\
\hline & 34,18 & MSB & & & & & 54,18 & SAC \\
\hline \multirow[t]{2}{*}{ Mituaçu } & 24,85 & INS & $S$ & $S$ & $S$ & $\mathrm{~N}$ & 73,18 & MSB \\
\hline & 8,09 & INS & & & & & 56,42 & SAC \\
\hline \multirow[t]{2}{*}{ Colinas do Sul } & 53,98 & MSB & $\mathrm{E}$ & $\mathrm{N}$ & $\mathrm{E}$ & $\mathrm{N}$ & 53,98 & MSB \\
\hline & 35,27 & MSB & & & & & 35,27 & MSB \\
\hline \multirow[t]{2}{*}{ Engenho Velho } & 31,81 & BSB & $S$ & $S$ & $S$ & $\mathrm{~N}$ & 80,14 & SAC \\
\hline & 8,20 & INS & & & & & 56,53 & SAC \\
\hline
\end{tabular}

${ }^{1)}$ INS - Insalubre; BSB - Baixa Salubridade; MSB - Média Salubridade; SAC - Salubridade Aceitável; SAL - Salubre. 
Silva, N. V. S.; Nascimento, R. Q.; Silva, T. C.

Tabela 8 - Resultados obtidos para a alocação de investimentos de 36,68\% do valor total, R\$2.740. I50,00; valores do ISA/JPI e I atuais e segundo a alocação dos benefícios priorizados propostos de acordo com o modelo MPIS/PL, com as respectivas situações de salubridade

\begin{tabular}{|c|c|c|c|c|c|c|c|c|}
\hline \multirow[t]{4}{*}{ Comunidades } & \multicolumn{2}{|c|}{ Atual } & \multicolumn{4}{|c|}{ Benefícios priorizados } & \multicolumn{2}{|c|}{ Proposta } \\
\hline & Valor & $\begin{array}{l}\text { Situação da } \\
\text { salubridade }\end{array}$ & SAA & SES & SRS & DU & Valor & $\begin{array}{l}\text { Situação da } \\
\text { Salubridade }\end{array}$ \\
\hline & ISA/JP1 & ISA/JP1 & & & & & ISA/JP1 & ISA/JP1 \\
\hline & I & I & & & & & I & I \\
\hline \multirow[t]{2}{*}{ Mumbaba de Baixo } & 55,17 & MSB & $\mathrm{E}$ & $S$ & $\mathrm{E}$ & S & 83,97 & SAC \\
\hline & 34,86 & MSB & & & & & 63,66 & SAL \\
\hline \multirow[t]{2}{*}{ Gramame } & 53,11 & MSB & $\mathrm{E}$ & $S$ & $\mathrm{E}$ & $\mathrm{N}$ & 73,11 & MSB \\
\hline & 34,18 & MSB & & & & & 54,18 & SAC \\
\hline \multirow[t]{2}{*}{ Mituaçu } & 24,85 & INS & $S$ & $S$ & $S$ & $S$ & 81,76 & SAC \\
\hline & 8,09 & INS & & & & & 65,00 & SAL \\
\hline \multirow[t]{2}{*}{ Colinas do Sul } & 53,98 & MSB & E & $S$ & E & $\mathrm{N}$ & 73,98 & MSB \\
\hline & 35,27 & MSB & & & & & 55,27 & SAC \\
\hline \multirow[t]{2}{*}{ Engenho Velho } & 31,81 & BSB & $S$ & $S$ & $S$ & $\mathrm{~N}$ & 80,14 & SAC \\
\hline & 8,20 & INS & & & & & 56,53 & SAC \\
\hline
\end{tabular}

${ }^{(1)}$ INS - Insalubre; BSB - Baixa Salubridade; MSB - Média Salubridade; SAC - Salubridade Aceitável; SAL - Salubre.

Tabela 9 - Resultados obtidos para a alocação de investimentos de 79,81\% do valor total, R\$ 5.962.576,00; valores do ISA/JPI e I atuais e segundo a alocação dos benefícios priorizados propostos de acordo com o modelo MPIS/PL, com as respectivas situações de salubridade

\begin{tabular}{|c|c|c|c|c|c|c|c|c|}
\hline \multirow[t]{4}{*}{ Comunidades } & \multicolumn{2}{|c|}{ Atual } & \multicolumn{4}{|c|}{ Benefícios Priorizados } & \multicolumn{2}{|c|}{ Proposta } \\
\hline & Valor & $\begin{array}{l}\text { Situação da } \\
\text { salubridade }\end{array}$ & SAA & SES & SRS & $\mathrm{DU}$ & Valor & $\begin{array}{l}\text { Situação da } \\
\text { salubridade }\end{array}$ \\
\hline & ISA/JP1 & ISA/JP1 & & & & & ISA/JP1 & ISA/JP1 \\
\hline & I & I & & & & & I & I \\
\hline \multirow[t]{2}{*}{ Mumbaba de Baixo } & 55,17 & MSB & $\mathrm{E}$ & $S$ & $\mathrm{E}$ & $S$ & 83,97 & SAC \\
\hline & 34,86 & MSB & & & & & 63,66 & SAL \\
\hline \multirow[t]{2}{*}{ Gramame } & 53,11 & MSB & $\mathrm{E}$ & S & $\mathrm{E}$ & S & 82,26 & SAC \\
\hline & 34,18 & MSB & & & & & 63,33 & SAL \\
\hline \multirow[t]{2}{*}{ Mituaçu } & 24,85 & INS & S & S & S & $\mathrm{N}$ & 73,18 & MSB \\
\hline & 8,09 & INS & & & & & 56,42 & SAC \\
\hline \multirow[t]{2}{*}{ Colinas do Sul } & 53,98 & MSB & $\mathrm{E}$ & S & $\mathrm{E}$ & $S$ & 82,38 & SAC \\
\hline & 35,27 & MSB & & & & & 63,67 & SAL \\
\hline \multirow[t]{2}{*}{ Engenho Velho } & 31,81 & BSB & S & S & S & $\mathrm{N}$ & 80,14 & SAC \\
\hline & 8,20 & INS & & & & & 56,53 & SAC \\
\hline
\end{tabular}

(1) INS - Insalubre; BSB - Baixa Salubridade; MSB - Média Salubridade; SAC - Salubridade Aceitável; SAL - Salubre. 
investimento presumido. Entretanto, apesar desta situação menos favorável, nesta comunidade ocorreria melhoria bastante positiva, de mais de $48 \%$ no valor do ISA/JP1, o que lhe permitiria sair da condição Insalubre. Com relação ao indicador I do saneamento básico, Mituaçu passaria à condição de Salubridade Aceitável.

Para o cenário resumido na Tabela 10 todos os benefícios atualmente ausentes nas comunidades seriam alocados. Mesmo assim, apesar desta grande evolução, nenhuma comunidade atingiria a situação Salubre, quanto à salubridade ambiental. No tocante ao indicador de saneamento básico, I, todos atingiriam a condição Salubre, conforme esperado, para $100 \%$ dos valores disponíveis para investimento. Ressalta-se, portanto, a evolução de Mituaçu e Engenho Velho, que na situação atual são as comunidades mais problemáticas em termos dos valores de ISA/JP1 e I, para o alcance da situação Salubridade Aceitável.

\section{CONCLUSÕES}

O objetivo do modelo MPIS/PL é oferecer as melhores alternativas de investimento, interpretadas como as que mais contribuem para o aumento da salubridade ambiental e da condição do saneamento básico, expressos nos valores dos indicadores ISA/JP1 e I, com os menores valores de investimento.

No desenvolvimento do modelo MPIS/PL, para alocação de investimentos em saneamento básico, que compreende serviços de abastecimento de água, esgotamento sanitário, limpeza urbana e manejo de resíduos sólidos, e drenagem e manejo de águas pluviais nos termos da Lei No $11.445 / 07$, estabeleceu-se uma função objetivo além de uma série de critérios multivariados. Objetivou-se a maximização de uma função correspondente ao somatório dos indicadores desses serviços, I, da função descritiva do ISA/JP1, ponderada pelos valores das populaçôes de cada comunidade em análise. As saídas do modelo são as variáveis de alocação 1 ou 0 , significando alocação ou não dos diversos benefícios em consideração.

No estudo de caso mostrado, é interessante destacar alguns resultados notáveis como o que aparece quando $36,68 \%$ do valor total são disponíveis para investimento e três das cinco comunidades alcançam a situação de Salubridade Aceitável, enquanto as outras passam a uma condição de Média Salubridade com relação à salubridade ambiental; cinco alcançam situações iguais ou superiores à condição Salubridade Aceitável referente ao indicador de saneamento básico.
O uso do modelo MPIS/PL mostra-se bem sucedido uma vez que oferece mais opções de investimentos de menor porte para mais benefícios em todas as comunidades analisadas, com respostas bastante positivas expressas nos indicadores ISA/JP1 e I para recursos financeiros baixos relativamente ao total dos valores orçados. Uma versão mais simplificada do modelo pode ser facilmente implementada apenas para o segmento saneamento básico, desconsiderando-se as variáveis complementares e com saídas dedicadas a este setor.

Deve-se ressaltar também que um ponto positivo na concepção do modelo está na versatilidade em oferecer aos gestores públicos um leque de opçôes visando o aumento da salubridade ambiental, que sejam compatíveis com os recursos disponíveis ou a serem disponibilizados pelas diversas instâncias de governo.

\section{AGRADECIMENTOS}

Os autores agradecem ao $\mathrm{CNPq}$ - Conselho Nacional de Desenvolvimento Científico e Tecnológico pelo financiamento da pesquisa "Desenvolvimento de um Sistema de Apoio à Decisão para a Gestão Urbana baseado em Indicadores Ambientais" - processo CNPq no 47400/2004-4, que teve an-

Tabela 10 - Resultados obtidos para a alocação de investimentos de 100\% do valor total, R\$7.4 I7।.003,00; valores do ISA/JP I e I atuais e segundo a alocação dos benefícios priorizados propostos de acordo com o modelo MPIS/PL, com as respectivas situações de salubridade

\begin{tabular}{|c|c|c|c|c|c|c|c|c|}
\hline \multirow[t]{4}{*}{ Comunidade } & \multicolumn{2}{|c|}{ Atual } & \multicolumn{4}{|c|}{ Benefícios Priorizados } & \multicolumn{2}{|c|}{ Proposta } \\
\hline & Valor & $\begin{array}{l}\text { Situação da } \\
\text { salubridade }\end{array}$ & SAA & SES & SRS & DU & Valor & $\begin{array}{l}\text { Situação da } \\
\text { salubridade }\end{array}$ \\
\hline & ISA/JP1 & ISA/JP1 & & & & & ISA/JP1 & ISA/JP1 \\
\hline & I & I & & & & & I & I \\
\hline \multirow[t]{2}{*}{ Mumbaba de Baixo } & 55,17 & MSB & $\mathrm{E}$ & $S$ & $\mathrm{E}$ & $S$ & 83,97 & SAC \\
\hline & 34,86 & MSB & & & & & 63,66 & SAL \\
\hline \multirow[t]{2}{*}{ Gramame } & 53,11 & MSB & $\mathrm{E}$ & S & $\mathrm{E}$ & S & 82,26 & SAC \\
\hline & 34,18 & MSB & & & & & 63,33 & SAL \\
\hline \multirow[t]{2}{*}{ Mituaçu } & 24,85 & INS & S & S & S & S & 81,76 & SAC \\
\hline & 8,09 & INS & & & & & 65,00 & SAL \\
\hline \multirow[t]{2}{*}{ Colinas do Sul } & 53,98 & MSB & $\mathrm{E}$ & $S$ & $\mathrm{E}$ & S & 82,38 & SAC \\
\hline & 35,27 & MSB & & & & & 63,67 & SAL \\
\hline \multirow[t]{2}{*}{ Engenho Velho } & 31,81 & BSB & $S$ & $S$ & S & S & 88,61 & SAC \\
\hline & 8,20 & INS & & & & & 65,00 & SAL \\
\hline
\end{tabular}

(1) INS - Insalubre; BSB - Baixa Salubridade; MSB - Média Salubridade; SAC - Salubridade Aceitável; SAL - Salubre. 
damento no Laboratório de Recursos Hídricos e Engenharia Ambiental - LARHENA, do Centro de Tecnologia da Universidade Federal da Paraíba. Agradecem ainda à Secretaria Executiva do Meio Ambiente da Prefeitura Municipal de João Pessoa, Paraíba, pelo apoio à realização deste trabalho.

\section{REFERÊNCIAS}

BATISTA, M. E. M; SILVA, T. C. O Modelo ISA/JP - Indicador de Performance para Diagnóstico do Saneamento Ambiental Urbano. Revista Engenharia Sanitária e Ambiental. Rio de Janeiro, v. 11, n. 1, p.62-71, Jan./Mar. 2006. Disponível em: http://www.abesdn.org.br/publicacoes/engenharia/resaonline/v11n01/ v1ln01a05.pdf. Acesso em: 02 set. 2007

BRASIL. FUNDAÇÃO NACIONAL DESAÚDE. Portaria n ${ }^{\circ}$ 151, de 20 Fevereiro de 2006. Diário Oficia da União, Brasília, DF, 21 de fev. 2006. Disponível em: http://www.funasa.gov.br/web\%20Funasa/ Legis/pdfs/portarias/Port_151_2006.pdf. Acesso em : 15 jan. 2006.

BRASIL. LEI No 11.445 de 5 de janeiro de 2007. Estabelece diretrizes nacionais para o saneamento básico; altera as Leis nos 6.766, de 19 de dezembro de 1979, 8.036, de 11 de maio de 1990, 8.666, de 21 de junho de 1993, 8.987, de 13 de fevereiro de 1995; revoga a Lei no 6.528, de 11 de maio de 1978; e da outras providências. Disponível em: http://www. planalto.gov.br/ccivil/_Ato2007-2010/2007/Lei/_ leis2007.htm. Acesso em: 06 set. 2007.
DIAS, M. C.; BORJA, P. C.; MORAES, L. R. S. Indice de Salubridade Ambiental em Áreas de Ocupação Espontâneas: Um Estudo em Salvador - Bahia. Engenharia Sanitária e Ambiental. Rio de Janeiro - RJ, v. 9, n.1, p. 82-92, Jan./Mar.2004. Disponível em: http:/www.abes-dn.org.br/publicacoes/en-genharia/resaonline/v9n1/v9n1t01. pdf. Acesso em: 02 set. 2007.

NEVES, C.; NEVES, M. S. Proposta Metodológica para Seleção e Hierarquização de Projetos de Saneamento. In: SIMPÓSIO EM ENGENHARIA DE PRODUÇÃO, 10, 2003, Bauru. Anais.... Bauru: UNESP, p. 1-10, 2003.

PRADO, D. S. Programação Linear. Série Pesquisa Operacional, 1. Belo Horizonte/MG. Editora de Desenvolvimento Gerencial, 208 p. 1999.

ROMERA e SILVA, P. A. Contribuição para o Estabelecimento de Metodologia de Suporte à Decisão em Políticas de Saneamento. 2000. 192 f. Tese (Doutorado em Geociência e Meio Ambiente) - Pós -Graduação em Geociência e meio Ambiente. Instituto de Geociências e Ciências Exatas, Universidade Estadual de Paulista, Rio Claro, 2000.

SÃO PAULO. In: ISA - Indicador de Salubridade Ambiental. Secretaria de Recursos Hídricos, Saneamento e Obras. Manual Básico. São Paul, Brasil, 37 p. 1999.

SILVA, N. V. S. da. As Condiçōes de Salubridade Ambiental das Comunidades Periurbanas da Bacia do Baixo Gramame: Diagnóstico e Proposição de Beneficios. 2006. 122 f. Dissertação (Mestrado em Engenharia Urbana). Universidade Federal da Paraíba, João Pessoa. Setembro, 2006.
TEIXEIRA, J. C.; HELLER, L. Modelo de Priorização de Investimentos em Saneamento com Ênfase em Indicadores de Saúde: Desenvolvimento e Aplicação em uma Companhia Estadual. Revista Engenharia Sanitária e Ambiental, Rio de Janeiro, v. 6, n 3-4, p.138-146, Jul/Set-Out/Dez, 2001. Disponível em: http://www.abes-dn.org.br/publicacoes/engenharia/posicoes/posicao2001.htm. Acesso em: 08 ago. 2006.

Priorização de Investimentos em Saneamento baseada em Indicadores Epidemiológicos e Financeiros. Revista Engenharia Sanitária e Ambiental, Rio de Janeiro, v.8, n 3, p.187-195, Jul./Set., 2003. Disponível em: http://www.abes-dn.org.br/publicacoes/engenharia/resaonline/v8n3/v8n3a05.pdf. Acesso em: 08 ago. 2006.

Endereço para correspondência:

Nayra Vicente Sousa da Silva

Rua Radialista Marcone

Altamirando, 34

58075-080 Ernesto Geisel - PB

- Brasil

Tel: (83) 32 I 6-7684

E-mail: nayravicent@yahoo.com.br 